\title{
ELECTRICAL ANHARMONICITY IN VIBRATING SYSTEMS CONTAINING MECHANICAL ANHARMONICITY DUE TO THE RELAXATION OF THE MOLECULAR ELECTRONIC CHARGE DISTRIBUTION
} JEREMY BURDETT * Department of Chemistry, University of Michigan, Ann .Arbor, Michigan 48104, USA

Received 20 January 1970

\begin{abstract}
The contributions of the relaxation of the molecular electronic charge distribution on vibration to the first and second molecular dipole moment derivatives with respect to a vibration coordinate are calculated.
\end{abstract}

Several investigations have correlated the shape of the molecular vibrational potential surface with the properties of the relaxation of the molecular electronic charge distribution on vibration [1,2]. In this communication we look at the contribution to the intensity of infra-red absorption bands from this source by calculating that part of the dipole moment due to the relaxation of the electronic distribution, on vibration, as a function of $S_{i}$, the $i$ th vibrational coordinate of the molecule.

We expand the electronic-hamiltonian in the usual way

$$
\mathscr{H}=\mathscr{H}^{0}+\mathscr{H}_{i} s_{i}+\frac{1}{2} \mathscr{H}_{i i} s_{i}^{2}+\ldots,
$$

where $\mathscr{H}^{\circ}, \mathscr{H}_{i}$ and $\mathscr{H}_{i i}$ are the, equilibrium hamiltonian and the first and second derivatives of the hamiltonian with respect to $S_{i}$, taken at the equilibrium position, respeciively. Treating the terms past $\mathscr{K}^{\mathrm{O}}$ as a perturbation $(\mathcal{O})$ for the ground electronic state of the system, the wave functional dependence on $S_{i}$ is given by:

$$
|0\rangle^{\prime}=|0\rangle-\Sigma^{\prime} \frac{V_{o m}}{E_{m}^{(0)}-E_{0}^{(0)}}|m\rangle
$$

where $|0\rangle, \ldots,|m\rangle$ represent the eigenstates of the equilibrium configuration. The contribution to the total dipole moment, as a function of $S_{i}$ is then given by:

$$
\mu_{\mathrm{oo}}^{\prime}=e\left\langle 0^{\prime}|r| 0^{\prime}\right\rangle=\mu_{\mathrm{oo}}-2 S_{i} \sum_{m}^{\prime} \frac{V_{\mathrm{o} m} \mu_{\mathrm{o} m}}{E_{m}^{(0)}-E_{0}^{(0)}}+S_{i}^{2} \sum_{k} \sum_{m}^{\prime} \frac{V_{\mathrm{o} k} V_{m \mathrm{O}^{\mu}} \mu_{k}}{\left(E_{k}^{(0)}-E_{0}^{(0)}\right)\left(E_{m}^{(0)}-E_{0}^{(0)}\right)} .
$$

On a two state model which is useful in treating vibrational force constants [2], the dipole moment correct to second order in $S_{i}$ is,

$$
\mu_{\mathrm{oO}}^{\prime}=\mu_{\mathrm{oO}}-2 S_{i} \frac{\left\langle 0\left|\mathscr{K}_{i}\right| n\right\rangle}{E_{n i}^{(0)}-E_{0}^{(0)}} \mu_{n \mathrm{O}}+S_{i}^{2}\left[\frac{\left|\left\langle 0\left|\mathscr{K}_{i}\right| n\right\rangle\right|^{2}}{\left(E_{n}^{(0)}-E_{0}^{(0)}\right)^{2}} \mu_{n n}-\frac{\left\langle 0\left|\mathscr{K}_{i i}\right| n\right\rangle}{E_{n}^{(0)}-E_{0}^{(0)}} \mu_{n 0}\right]+O\left(S_{i}^{3}\right) .
$$

We can identify these terms with those appearing in the series expansion of the dipole moment,

* Fellow of the Power Foundation. 


$$
\mu=\mu_{0}+\left(\frac{\partial \mu}{\partial S_{i}}\right)_{0} S_{i}+\frac{1}{2}\left(\frac{\partial^{2} \mu}{\partial s_{i}^{2}}\right)_{0} S_{i}^{2}+\ldots
$$

Each term in eq. (4) makes a contribution to the corresponding factor in eq. (5).

The contribution to the intensity of an infra-red fundamental by this process is then given by *:

$$
\frac{8 \pi^{3} N \nu_{i}}{3 c h}\left|\left\langle v^{\prime}\left|s_{i}\right| v^{\prime \prime}\right\rangle\right|^{2} \frac{4\left|\left\langle 0\left|\mathscr{H}_{i}\right| \eta\right\rangle\right|^{2}}{\left(E_{n}^{(0)}-E_{0}^{(0)}\right)^{2}} \mu_{n o}^{2} .
$$

Hence in vibrational modes where the effect of electronic relaxation is important (i.e. $\left\langle 0\left|\mathscr{K}_{i}\right| n\right\rangle ;\left[E_{n}^{(\mathrm{o})}-E_{0}^{(\mathrm{o})}\right]$ is large), a correspondingly large contribution to the fundamental intensity by this mechanism is expected. For example, the shape of the potential surface of the out-of-plane bending motion $\left(S_{2}\right)$ of the methyl radical $\mathrm{CH}_{3}$ has been suggested [2] to be largely determined by the relaxation of the electronic charge distribution. Here the transition density is located along the three-fold axis of the planar radical and $v_{2}$ is the only vibrational mode intense enough to be observed [4]. In $\mathrm{CCl}_{3}$, which is pyramidal, the corresponding vibration has not been observed [5]. As the deviation from planarity of a $\mathrm{CX}_{3}$ species increases, the energy separation $E_{n 2}^{(0)}-E_{0}^{(0)}$ increases rapidly as may be seen from a Walsh diagram [6]. The electron relaxation contribution to the force constant in $S_{2}$ drops sharply, as has been discussed previously [2]. Hence the electron relaxation term in eq. (6) is expected to drop very sharply as the deviation from planarity increases. When contrasting the non-observation of $\nu_{2}$ in the literature infrared spectrum of $\mathrm{CCl}_{3}$, but its appearence in the $\mathrm{CH}_{3}$ spectrum, this effect may be the all-important factor.

The intensity of the first overtone may be approached using the same recipe. Here, using the first order wavefunction, we examine the properties of the term

$$
\left[\frac{\mid\left\langle\left. 0\left|\mathscr{K}_{i}\right| n\right|^{2}\right.}{E_{n}^{(0)}-E_{0}^{(0)}} \mu_{m 2}-\frac{\left\langle 0\left|\mathscr{K}_{i i}\right| n\right\rangle}{E_{n}^{(0)}-E_{0}^{(0)}} \mu_{n o}\right]^{2}
$$

For the centrosymmetric methyl radical $\mu_{n n}=0$ and $\left\langle 0\left|\mathscr{K}_{i i}\right| n\right\rangle=0$ by symmetry $\left(S_{2} \rightarrow a_{2}^{\prime \prime}\right)$. Hence this entire term is zero. It is then not surprising that no first overtone of $\nu_{2}$ was observed in an infra-red study [4].

Another series of systems in which electron relaxation effects are believed to be important are diazomethane and ketene [2]. Here the rocking and wagging force constants of the $\mathrm{CH}_{2}$ group are extremely low and the intensity of both the fundamentals and first overtones of these modes are extremely high $[7,8]$. The high intensity of the fundamentals follows from the discussion above for the $\mathrm{CH}_{3}$ radical. The high intensity of the first overtone follows from the size of the parameter ( 7$)$. Here $\left\langle 0\left|\mathscr{K}_{i i}\right| n\right\rangle=0$ since neither the rocking or wagging modes are totally symmetric, but $\mu_{n n} \neq 0$ due to the lack of a center of symmetry. $\left.\left.\langle 0| \mathscr{H}_{i}\right] n\right\rangle /\left[E_{n}^{(0)}-E_{0}^{(o)}\right]$ is large [2]. Hence we may expect a large contribution to $\left(\partial^{2} \mu / \tilde{C} S_{i}^{2}\right)_{0}$ from the relaxation of the electrons, leading to high intensities of the first overtones.

In the above argument we have only considered the magnitude of the dipole moment terms. For systems containing large contributions to vibrational force constants from the electron relaxation terms, large anharmonic constants seem to be the rule [2]. There will then be some contribution to the infrared intensity from the terms $\left|\left\langle v^{\prime}\left|S_{i}^{x}\right| v^{\prime \prime}\right\rangle\right|^{2}$ arising from the breakdown of the harmonic oscillator approximation.

In a similar fashion we may examine higher terms of eq. (5) by taking higher terms into account in eq. (1) and by using a wavefunction correct to second order. If this latter improvement is included (7) becomes more complex

$$
\left[\left(\mu_{n n}-\mu_{00}\right) \frac{\left|\left\langle 0\left|\mathscr{K}_{i}\right| n\right\rangle\right|^{2}}{\left(E_{n}^{(0)}-E_{0}^{(0)}\right)^{2}}+\mu_{0 n} \frac{\left\langle 0\left|\mathcal{H}_{i}\right| n\right\rangle}{\left(E_{n}^{(0)}-E_{0}^{(0)}\right)^{2}}\left[\left\langle n\left|\mathscr{F}_{i}\right| n\right\rangle-\left\langle 0\left|\mathscr{K}_{i}\right| 0\right\rangle\right]+\mu_{\mathrm{o} n} \frac{\left\langle 0\left|\mathcal{X}_{i i}\right| n\right\rangle}{E_{n}^{(0)}-E_{0}^{(0)}}\right]^{2}
$$

* See for example ref. [3]. 
the same considerations discussed above still apply, and the overall result is similar.

To conclude therefore, we may say that in systems where there is a significant pseudo Jahn-Teller softening of the $i$ th vibrational force constant, we can expect a non-trivial electrical anharmonicity, as shown in this communication, matched by the mechanical anharmonicity investigated in ref. [2], in this $i$ th mode.

\section{REFERENCES}

[1] R.F.W. Bader, Mol. Phys. 3 (1960) 137.

[2] J.K. Bilcdett, J.Chem. Phys., to be published.

[3] G. Herzberg, NIolecular spectra and molecular structure II. Infra-red and Raman spectra (Van Nostrand, Princeton, 1945) p. 261.

[4] M.E.Jacox and D.E.Milligan, J.Chem. Phys. 47 (1967) 5146.

[5] L. Andrew's, J.Chem. Phys. 48 (1968) 972.

[G] A.D.Walsh, J.Chem. Soc. (1953) 2260.

[7] C.B. Moore and G.C.Pimentel, J.Chem. Phys. 40 (1964) 329, 342.

[8] C.B.Moore and G.C.Pimentel, J.Chem. Phys. 38 (1963) 2816. 